\title{
Batch service Queuing system with Phase of service and various vacation policies
}

\author{
S. Maragathasundari, Associate professor, Department of Mathematics, Kalasalingam Academy of
}

Research and Education. Krishnankoil, India. maragatham01@gmail.com.

K.Kavitha, Assistant professor, Department of Mathematics, Velammal Instititute of Technology,

Chennai, India. kavibiet@gmail.com

Abstract This paper considers a $\mathrm{M}^{\mathrm{x}} / \mathrm{G} / 1$ line with arbitrary breakdowns and various vacation. Service is given in phases. After the fulfilment of service,the server goes for a get-away of irregular span and subsequent to coming back from get-away, the server chooses to take an all-encompassing get-away which is optional. A idea of remain by server is presented during the hour of stretched out vacation. The server may expose to separate and it get into a fix procedure quickly with no delay. We treat Reneging in this paper when the server is under repair. Arrival of clients follows a Poisson distribution. All different parameters follow general distribution. Steady state consequences of the lining model and all the execution measures are determined. Numerical outline and graphical methodology justifies the model.

Keywords - Optional Extended Vacation, Phases of service, Reneging and Standby server

\section{INTRODUCTION}

There is a colossal writing in the investigation of Queuing speculation, because of its wide congruity in showing over blockage issues. The Queuing model with server vacation has been particularly mulled over and adequately applied in various areas. Here Vacation implies the transitory unavailability of organizations in the structure .B. Balamurugan and S. Maragathasundari [1], made a study on the performance analysis of a batch arrival queue with two stages of service . Dhanalakshmi and S. Maragathasundari [2], studied Mobile adhoc networks problem through queuing approach. P. Karunakaran and $\mathrm{S}$. Maragathasundari [3], made a Analysis on Queuing System with Setup Time in Revamp Process . Kailash C. Madan and Gautam Choudhury[4] derived the steady results for a M /G/1 Queue with a Bernoulli vacation schedule under restricted admissibility policy .S. Maragathasundari, et.al[5] determined the execution measures for a Non Markovian Queuing System with Restricted Admissibility.An analysis was carried out in M/G/1 feedback queue with three stage and multiple server vacation by . S. Maragathasundari and S. Srinivasan[6]. S. Maragathasundari and S. Srinivasan[7] studied the concept of reneging a Non-Markovian Multistage Batch arrival queue with breakdown and reneging. S. Maragathasundari and S. Radha[8], investigated the mathematical modelling in Non markovian Queue.S.Maragathasundari and K.Karthikeyan[9],made a analysis report on Queuing Vacation with optional stages of service .Monita Baruah et.al[10] explained about the Balking and Re-service in a vacation Queue with Batch Arrival and Two Types of Heterogeneous Service. S.
Radha, S. Maragathasundari, S.Sowmiyah and S. Kishore Eswar[11] examined a Queuing Classification in NonDestructive Testing. An MX/G/1 queue with randomized working vacations and at most $\mathbf{J}$ vacations is well defined by Shan Gao and Yunfei Yao[12]. S. Sowmiah and S. Maragathasundari[13] studied the performance measure of bulk input queue with $\mathrm{N}$ type of additional optional service, service interruption and deterministic vacation P. Suthersan and S. MaragathaSundari[14]investigated the Queuing Analysis on Multiple Vacation Policies .

\section{ALGEBRAIC REPRESENTATION OF THE MODEL}

Customers arrival follows a poisson distribution with arrival rate $\lambda_{t}$. Service is provided in phases.All the other parameters follows a general distribution.Break down arrival rate is $\gamma>0$.

Let $A(x)$ and $a(x)$, be the distribution function and the density function of phase service

Let $\psi_{i}(x)$ be the conditional probability of a completion of phase of service and it is given by

$\psi_{i}(x)=\frac{a(x)}{1-A(x)}, a(x)=\psi_{i}(x) e^{-\int_{0}^{x} \psi_{i}(t) d t}$

Similary, the process is repeated for setup time,compulsory vacation, optional extended vacation, Repair process. Hence we have the following: 
In case of compulsory vacation $S_{n}$, we have $\psi_{s}(x)=\frac{c(x)}{1-c(x)}, c(x)=\psi_{s}(x) e^{-\int_{0}^{x} \psi_{s}(t) d t}$

After the completion of compulsory vacation, with probability $r$ the server takes a optional extended vacation.

For the optional extended vacation $M_{n}$ : $\psi_{e}(x)=\frac{d x)}{1-D(x)}$,

$d(x)=\psi_{e}(x) e^{-\int_{0}^{x} \psi_{\theta}(t) d t}$.During the time of optional extended vacation, a standby server is provided with parameter $\beta$.

For the Setup time $A_{n}: \psi_{a}(x)=\frac{b(x)}{1-B(x)}$, $b(x)=\psi_{a}(x) e^{-\int_{0}^{x} \psi_{a}(t) d t}$

For the Repair process $K_{n}: \quad \psi_{k}(x)=\frac{\epsilon(x)}{1-E(x)}$, $e(x)=\psi_{k}(x) e^{-\int_{0}^{x} \psi_{k}(t) d t}$

The concept of Reneging follows exponential distribution with parameter $\xi$ and $y(t)=\xi e^{-\xi t} d t, \quad \xi>0$ and it occurs during repair process.

\section{GOVERNING EQUATIONS OF THE MODEL}

To start with, first let us frame the governing equations of the queuing model defined.

$\frac{\partial}{\partial x} T_{n}^{(i)}(x)+\left(\lambda_{t}+\psi_{i}(x)+\gamma\right) T_{n}^{(i)}(x)=$

$\lambda_{t} \sum_{r=1}^{n} d_{r} T_{n-r}^{(i)}(x)$,

$\frac{\partial}{\partial x} T_{0}^{(i)}(x)+\left(\lambda_{t}+\psi_{i}(x)+\gamma\right) T_{0}^{(i)}(x)=0$.

(2)

$\frac{\partial}{\partial x} A_{n}(x)+\left(\lambda_{t}+\psi_{\alpha}(x)\right) A_{n}(x)=$

$\lambda_{t} \sum_{r=1}^{n} d_{r} A_{n-r}(x)$.

$\frac{\partial}{\partial x} A_{0}(x)+\left(\lambda_{t}+\psi_{a}(x)\right) A_{0}(x)=0$.

(4)

$\frac{\partial}{\partial x} S_{n}(x)+\left(\lambda_{t}+\psi_{s}(x)\right) S_{n}(x)=$

$\lambda_{t} \sum_{r=1}^{n} d_{r} S_{n-r}(x)$.

$\frac{\partial}{\partial x} S_{0}(x)+\left(\lambda_{t}+\psi_{s}(x)\right) S_{0}(x)=0$.

(6)

$\frac{\partial}{\partial x} M_{n}(x)+\left(\lambda_{t}+\psi_{\theta}(x)+\beta\right) M_{n}(x)=$

$\lambda_{t} \sum_{r=1}^{n} d_{r} M_{n-r}(x)+\beta M_{n+1}(x)$.

(7)

$\frac{\partial}{\partial x} M_{0}(x)+\left(\lambda_{t}+\psi_{e}(x)+\beta\right) M_{0}(x)=\beta M_{1}(x)$.

(8) $\frac{\partial}{\partial x} K_{n}(x)+\left(\lambda_{t}+\psi_{k}(x)+\xi\right) K_{n}(x)=$

$\lambda_{t} \sum_{r=1}^{n} d_{r} K_{n-r}(x)+\xi K_{n+1}(x)$.

(9)

$\frac{\partial}{\partial x} K_{0}(x)+\left(\lambda_{t}+\psi_{k}(x)+\xi\right) K_{0}(x)=\xi K_{1}(x)$.

(10)

$\lambda_{t} C=$

$\int_{0}^{\infty} K_{0}(x) \psi_{k}(x) d x+\int_{0}^{\infty} M_{0}(x) \psi_{\theta}(x) d x+(1-$ r) $\int_{0}^{\infty} S_{0}(x) \psi_{s}(x) d x$.

(11)

Initial and boundary conditions:

$T_{n}^{(i)}(0)=$

$\int_{0}^{\infty} K_{n+1}(x) \psi_{k}(x) d x+\int_{0}^{\infty} M_{n+1}(x) \psi_{\theta}(x) d x+$

$(1-r) \int_{0}^{\infty} S_{n+1}(x) \psi_{s}(x) d x+\lambda_{t} D_{n+1} C$.

$A_{n}(0)=\int_{0}^{\infty} T_{n}^{(i)}(x) \psi_{i}(x) d x$.

(13)

$S_{n}(0)=\int_{0}^{\infty} A_{n}(x) \psi_{a}(x) d x$.

$M_{n}(0)=r \int_{0}^{\infty} S_{n}(x) \psi_{s}(x) d x$.

$K_{n}(0)=\gamma \int_{0}^{\infty} T_{n}^{(i)}(x) d x$.

(16).

\section{APPROACH OF SUPPLEMENTARY} VARIABLE METHOD

Apply the Concept of Supplementary variable method for the above set of equations

$$
\begin{aligned}
& \frac{\partial}{\partial x} T_{q}^{(i)}(x, z)+\left(\lambda_{t}-\lambda_{t} D(z)+\psi_{i}(x)+\right. \\
& \beta) T_{q}^{(i)}(x, z)=0 .
\end{aligned}
$$

$\frac{\partial}{\partial x} A_{q}(x, z)+\left(\lambda_{t}-\lambda_{t} D(z)+\psi_{a}(x)\right) A_{q}(x, z)=$ 0 .

$\frac{\partial}{\partial x} S_{q}(x, z)+\left(\lambda_{t}-\lambda_{t} D(z)+\psi_{s}(x)\right) S_{q}(x, z)=$ 0 .

$\frac{\partial}{\partial x} M_{q}(x, z)+\left(\lambda_{t}-\lambda_{t} D(z)+\psi_{\theta}(x)+\beta-\right.$

$\left.\frac{\beta}{z}\right) M_{q}(x, z)=0$.

$\frac{\partial}{\partial x} K_{q}(x, z)+\left(\lambda_{t}-\lambda_{t} D(z)+\psi_{k}(x)+\xi-\right.$ $\left.\frac{\xi}{z}\right) K_{q}(x, z)=0$. 
$z T_{q}^{(i)}(0, z)=\int_{0}^{\infty} K_{q}(x, z) \psi_{k}(x) d x+$

$\int_{0}^{\infty} M_{q}(x, z) \psi_{\theta}(x) d x+(1-$

r) $\int_{0}^{\infty} S_{q}(x, z) \psi_{s}(x) d x+c \lambda_{t}[D(z)-1]$.

$A_{q}(0, z)=\int_{0}^{\infty} T_{q}^{(i)}(x, z) \psi_{i}(x) d x$.

(23)

$S_{q}(0, z)=\int_{0}^{\infty} A_{q}(x, z) \psi_{a}(x) d x$.

(24)

$M_{q}(0, z)=r \int_{0}^{\infty} S_{q}(x, z) \psi_{s}(x) d x$.

$K_{q}(0, z)=\gamma z T_{q}^{(i)}(z)$. (26)

Integrating (11) from 0 to $x_{y}$ we obtain

$T_{q}^{(i)}(x, z)=T_{q}^{(i)}(0, z) e^{-\left(\lambda_{t}-\lambda_{t} D(z)+\beta\right)-\int_{0}^{x} \psi_{i}(t) d t}$.

(27)

Integrating (27) by parts, we get

$T_{q}^{(i)}(z)=T_{q}^{(i)}(0, z)\left[\frac{1-\bar{B}(m)}{m}\right]$.

(28)

Multiply (27) by $\psi_{i}(x)$ on both the sides and integrating over $\boldsymbol{x}_{y}$ we get

$\int_{0}^{\infty} T_{q}^{(i)}(x, z) \psi_{i}(x) d x=T_{q}^{(i)}(0, z) \bar{B}(m)_{s}$ where

$m=\lambda_{t}-\lambda_{t} D(z)+\beta$.

Repeating the same procedure, we get

$A_{q}(z)=A_{q}(0, z)\left[\frac{1-E(l)}{l}\right]$, where $l=\lambda_{t}-\lambda_{t} D(z)$.

(30)

$\int_{0}^{\infty} A_{q}(x, z) \psi_{a}(x) d x=A_{q}(0, z) \bar{B}(l)=$ $T_{q}^{(i)}(0, z) \bar{B}(m) \bar{B}(l)$.

$S_{q}(z)=S_{q}(0, z)\left[\frac{1-C(l)}{l}\right]$.

$\int_{0}^{\infty} S_{q}(x, z) \psi_{s}(x) d x=T_{q}^{(i)}(0, z) \bar{B}(m) \bar{B}(l) \bar{C}(l)$.

(33)

$M_{q}(z)=M_{q}(0, z)\left[\frac{1-\bar{D}(p)}{p}\right]$, where

$p=\lambda_{t}-\lambda_{t} D(z)+\beta-\frac{\beta}{z}$.

$\int_{0}^{\infty} M_{q}(x, z) \psi_{\theta}(x) d x=$

$r T_{q}^{(i)}(0, z) \bar{B}(m) \bar{B}(l) \bar{C}(l) \bar{D}(p)$.

$K_{q}(z)=K_{q}(0, z)\left[\frac{1-E(w)}{w}\right]$, where

$w=\lambda_{t}-\lambda_{t} D(z)+\xi-\frac{\xi}{z}$.

(36)

$\int_{0}^{\infty} K_{q}(x, z) \psi_{k}(x) d x=$

$\gamma z T_{q}^{(i)}(0, z)\left[\frac{1-\bar{B}(m)}{m}\right] \bar{E}(w)$.

(37)
Substituting (33), (35) and (37) in (22), we get

$T_{q}^{(i)}(0, z)=$

$C \lambda_{t}[D(z)-1]$

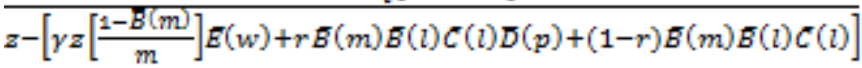
. (38)

\section{PROBABILITY GENERATING QUEUE SIZE, IDLE TIME AND UTILIZATION FACTOR}

Next in finding the probability generating function of the queue size, we get

$R_{q}(z)=T_{q}^{(i)}(z)+A_{q}(z)+S_{q}(z)+M_{q}(z)+$ $K_{q}(z)$.

(39)

$=$

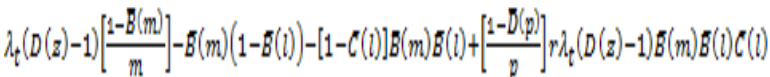

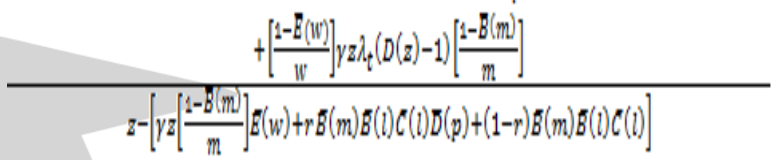

(40)

To find idle time we use the normalization condition

$R_{q}(z)+Q=1$, At $z=1, R_{q}(z)$ attains indeterminate form.

Hence, by the usage of L'Hopital's rule, we get

$\lim _{z \rightarrow 1} R_{q}(z)=R_{q}(1)=\frac{N^{\prime}(1)}{D^{\prime}(1)}$

Now from the above idle time $Q$ is given by

$Q=\frac{D^{\prime}(1)}{N^{\prime}(1)+D^{\prime}(1)}$

By condition $\rho=1-Q$, utilization factor is determined.

\section{PERFORMANCE MEASURES OF THE QUEUING SYSTEM}

To find the steady state average queue length, $L_{q}$, we adopt the following method

$L_{q}=\frac{d}{d z} R_{q}(z) \mathrm{at} z=1$

This attains indeterminate form $\frac{0}{0}$. Consider (39) as

$R_{q}(z)=\frac{N(z)}{D(z)}$

$N(z)$ and $D(z)$ are the numerator and denominator of the

R.H.S. of (40)

Apply L'Hopital's rule twice on (40) we obtain

$L_{\mathrm{q}}=\lim _{z \rightarrow 1} \frac{D^{\prime}(z) N^{\prime \prime}(\mathrm{z})-\mathrm{D}^{\prime \prime}(z) N^{\prime}(z)}{2\left(D^{\prime}(z)\right)^{2}}$

$=\frac{D^{\prime}(1) N^{\prime \prime}(1)-\mathrm{D}^{\prime \prime}(1) N^{\prime}(1)}{2\left(D^{\prime}(1)\right)^{2}}$ 


$$
\begin{aligned}
& D^{\prime}(1)=1-\left[\gamma\left(\left[\frac{1-\bar{B}(\gamma)}{\gamma}\right]+\left(-\bar{B}^{\prime}(\gamma)\right)+\left[\frac{1-\bar{B}(\gamma)}{\gamma}\right] E((V))\left(\lambda_{t}-\xi\right)\right)+\left\{-\bar{B}^{\prime}(\gamma) \lambda_{t}+\lambda_{t} E(B) \bar{B}(\gamma)+\lambda_{t} E(C) \bar{B}(\gamma)\right\}\right. \\
& +\bar{B}(\gamma)\left[r E(D)\left(\lambda_{t}-\beta\right)\right] \\
& D^{\prime}(1)=-\gamma\left\{2\left[\frac{1-\bar{B}(\gamma)}{\gamma}\right] E(W)\left\{\left(-\lambda_{t}+2 \xi\right)\right\}+\bar{B}^{-1}(\gamma) \lambda_{t}+\left[\frac{1-\bar{B}(\gamma)}{\gamma}\right] E\left(W^{2}\right)\left(-\lambda_{t}+\xi\right)^{2}\right. \\
& \left.+2\left(-\bar{B}^{\prime}(\gamma)\right)\left[E(w)\left(-\lambda_{t}+\xi\right)+1\right]\right\}-\lambda_{t}^{2}\left[E\left(B^{2}\right)+\bar{B}(\gamma)\left\{E\left(B^{2}\right)+E\left(C^{2}\right)+E(B) E(C)\right]\right. \\
& -\left[-\lambda_{t} \bar{B}^{\prime}(\gamma)+\lambda_{t} \bar{B}(\gamma)\{E(B)+E(C)\}\right] r E(D)\left(\lambda_{t}-\beta\right)-r E\left(D^{2}\right)\left(-\lambda_{t}+\beta\right)^{2} \bar{B}(\gamma) \\
& -\left[r E(D)\left(\lambda_{t}-\beta\right)\right]\left[\bar{B}^{\prime}(\gamma)+\lambda_{t} \bar{B}(\gamma)[E(B)+E(C)]\right] \\
& N^{\prime}(1)=\lambda_{t}\left[\frac{1-\bar{B}(\gamma)}{\gamma}\right]-\lambda_{t} \bar{B}(\gamma) E(B)-\bar{B}(\gamma) \lambda_{t} E(C)+\lambda_{t} \bar{B}(\gamma)[E(B)+E(C)] \\
& N^{\prime}(1)=\lambda_{t}\left[\frac{1-\bar{B}(\gamma)}{\gamma}-\bar{B}^{\prime}(\gamma)\right]-2 \bar{B}^{\prime}(\gamma) \lambda_{t}^{2} E(B)+\lambda_{t}^{2} \bar{B}(\gamma)\left(E\left(B^{2}\right)+E\left(C^{2}\right)+2 E(B) E(C)\right)-2 \bar{B}^{\prime}(\gamma) E(C) \lambda_{t}{ }^{2} \\
& +E(D)\left(-\lambda_{t}+\beta\right) r \lambda_{t} \bar{B}(\gamma)+r \lambda_{t} E(D) \bar{B}(\gamma)+\beta \lambda_{t} E(W)\left[\left(-\lambda_{t}+\beta\right)+1\right]\left[\frac{1-\bar{B}(\gamma)}{\gamma}\right] \\
& +\bar{B}^{\prime}(\gamma) \lambda_{t}^{2}[E(B)+E(C)]+\bar{B}(\gamma) \lambda_{t}^{2}\left[E\left(B^{2}\right)+E\left(C^{2}\right)\right]
\end{aligned}
$$

The other Queue performance measures are obtained using the Little's law.

\section{NUMERICAL ILLUSTRATION}

Consider, $\lambda_{t}=3.5, \psi_{\mathrm{i}}=3, \psi_{a}=3.7, \psi_{\mathrm{e}}=4, \psi_{\mathrm{s}}=5$, $\psi_{k}=6, r=0.5$,

$\bar{B}(\gamma)=\frac{\varphi_{i}}{\psi_{i}+\gamma}, \bar{B}^{\prime}(\gamma)=\frac{-\psi_{i}}{\left(\varphi_{i}+\gamma\right)^{2}}, E(B)=\frac{1}{\varphi_{a}}, E(C)=\frac{1}{\psi_{k}}$, $E(D)=\frac{1}{\psi_{\mathrm{s}}}, E(W)=\frac{1}{\psi_{k}}, E\left(B^{2}\right)=\frac{2}{\psi_{\mathrm{a}}^{2}}, E\left(C^{2}\right)=\frac{2}{\psi_{\mathrm{z}}^{2}}$, $E\left(D^{2}\right)=\frac{2}{\psi_{k}^{2}}, E\left(W^{2}\right)=\frac{2}{\psi_{k}^{2}}$,

\begin{tabular}{|c|c|c|c|c|c|}
\hline$Q$ & $\rho$ & $L_{\mathrm{q}}$ & $L$ & $W_{\mathrm{q}}$ & $W$ \\
\hline 0.6318 & 0.3682 & 1.3434 & 1.7116 & 0.4478 & 0.5705 \\
\hline 0.6305 & 0.3695 & 1.2015 & 1.5709 & 0.4005 & 0.5237 \\
\hline 0.6298 & 0.3702 & 1.0795 & 1.4497 & 0.3598 & 0.4832 \\
\hline 0.6294 & 0.3706 & 0.9688 & 1.3394 & 0.3229 & 0.4465 \\
\hline 0.6292 & 0.3708 & 0.8648 & 1.2356 & 0.2883 & 0.4119 \\
\hline
\end{tabular}

(i) The Effect of change of $\gamma=2,3,4,5,6$

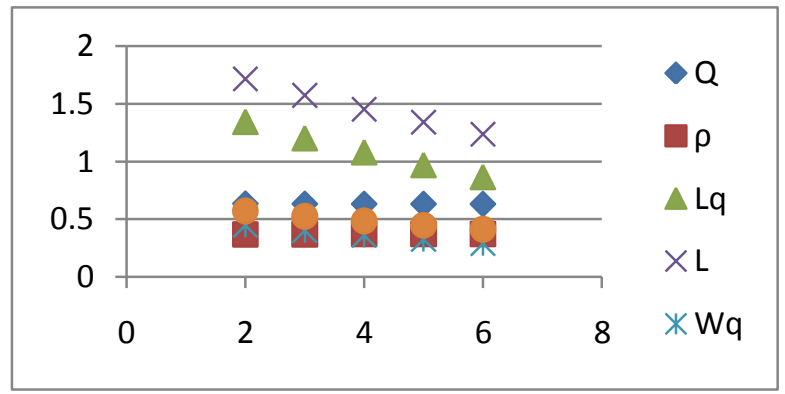

Fig.1 The variation of $\gamma$

As the break down increases, due to the concept of reneging, length of the queue and all the other performance measures gets decreased. (ii) The Effect of change of $\xi=3.4,3.9,4.4,4.9,5.4$

\begin{tabular}{|c|c|c|c|c|c|}
\hline$Q$ & $\rho$ & $L_{q}$ & $L$ & $W_{q}$ & $W$ \\
\hline 0.6318 & 0.3682 & 1.3434 & 1.7116 & 0.3838 & 0.4890 \\
\hline 0.6252 & 0.3748 & 1.2866 & 1.6614 & 0.3676 & 0.4747 \\
\hline 0.6184 & 0.3816 & 1.2223 & 1.6039 & 0.3492 & 0.4583 \\
\hline 0.6114 & 0.3886 & 1.1493 & 1.5379 & 0.3284 & 0.4394 \\
\hline 0.6041 & 0.3959 & 1.0664 & 1.4623 & 0.3047 & 0.4178 \\
\hline
\end{tabular}

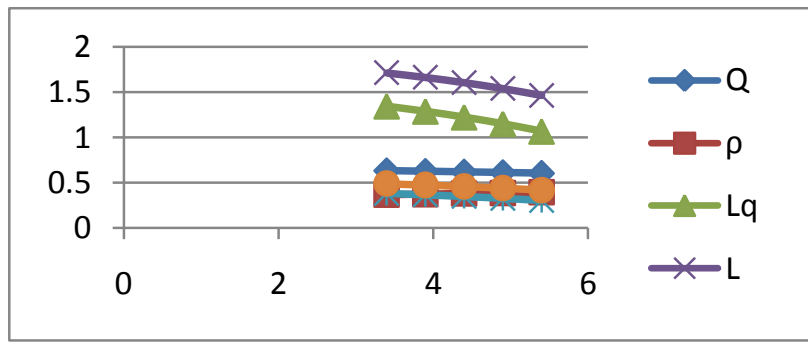

Fig.2 The variation of $\xi$.

As the reneging increases, all the performance measures gets reduced.

\section{VIII . CONCLUSION}

This paper deals with the queuing system with various parameters like phases of service, setup time, vacation, optional extended vacation and repair process. The corresponding probability generating function of the queue size and all the other execution measures are derived .Moreover the model is well rationalized with numerical analysis and graphical portrayal..The model can be extended by incorporating the ideas of stages of service, balking and stages of service. This model has wide applications in manufacturing industries , web designing, communication etc.,

\section{REFERENCES}

[1] B. Balamurugan and S. Maragathasundari, "A study on the performance analysis of a batch arrival queue with two stages of service Bernoulli schedule vacation extended vacation and service interruption", International journal of computer applications, Vol.124, No.12, pp.33-37, 2015.

[2] Dhanalakshmi and S. Maragathasundari ,"Mobile adhoc networks problem- A queuing approach", International journal of communication networks and distributed systems, vol.21, No.4, 2018.

[3] P. Karunakaran, S. Maragathasundari, A Analysis on Queuing System with Setup Time in Revamp Process , International Journal of Engineering and Advanced Technology (IJEAT) ISSN: 2249 - 8958, Volume-9 Issue-1S4, December 2019,967-969

[4] Kailash C. Madan and Gautam Choudhury , “ AnM /G/1 Queue with a Bernoulli vacation schedule under 
restricted admissibility policy", The Indian Journal of Statistics, Vol. 66, No. 1. pp. 175-193, 2004.

[5] S. Maragathasundari, R. S. Somasundaram, S. Radha, Non Markovian Queuing System with Restricted Admissibility Method ,International Journal of Engineering and Advanced Technology (IJEAT) ISSN: 2249 - 8958, Volume-9 Issue-1S4, December 2019,957-960

[6] S. Maragathasundari and S. Srinivasan, "Analysis of M/G/1 feedback queue with three stage and multiple server vacation", Applied mathematical sciences, Vol..6, No.125, Pp.6221-6240, 2012.

[7] S. Maragathasundari and S. Srinivasan , "A NonMarkovian Multistage Batch arrival queue with breakdown and reneging", Mathematical problems in engineering, Volume 2014/16 pages/ Article ID 519579/ http: // dx. Doi. Org / 10.1155/2014/ 519579. 2015.

[8] S. Maragathasundari and S. Radha,A study on the investigation of mathematical modelling in Non markovian Queue,Recent trends in pure and applied mathematics, AIP conference proceedings 2177,020042(2019):https://doi.org/10.1063/1.5135217, 2019,1-9

[9] S.Maragathasundari, K.Karthikeyan,Analysis report on Queuing Vacation with optional stages of service, International journal of research and analytical reviews,2019, Vol 6, issue 2, 283-290

[10] Monita Baruah, Kailash C. Madan and Tillal Eldabi ,“ Balking and Re-service in a vacation Queue with Batch Arrival and Two Types of Heterogeneous Service", Journal of Mathematics Resesarch, Vol. 4, No. 4, pp. 115-124, 2012.

[11]S. Radha, S. Maragathasundari, S.Sowmiyah, S. Kishore Eswar An Examination on Queuing Classification in Non-Destructive Testing , International Journal of Engineering and Advanced Technology (IJEAT) ISSN: 2249 - 8958, Volume-9 Issue-1S4, December 2019,669-674

[12] Shan Gao and Yunfei Yao , "An MX/G/1 queue with randomized working vacations and at most $\mathrm{J}$ vacations", International Journal of Computer Mathematics, Vol. 91, No. 3, pp. 368-383, 2014.

[13] S. Sowmiah and S. Maragathasundari , "A study on the analysis of performance measure of bulk input queue with $\mathrm{N}$ type of additional optional service, service interruption and deterministic vacation", Imperial Journal of Interdisciplinary Research, Vol.2, No.8, pp.1436-1444, 2016.
[14]P. Suthersan, S. MaragathaSundari, Queuing Analysis on Multiple Vacation Policies and Reneging , International Journal of Engineering and Advanced Technology (IJEAT) ISSN: 2249 - 8958, Volume-9 Issue-1S4, December 2019,948-951. 